\section{Double-balloon enteroscope-assisted enteral stent placement for malignant afferent-loop obstruction after Roux-en-Y reconstruction}
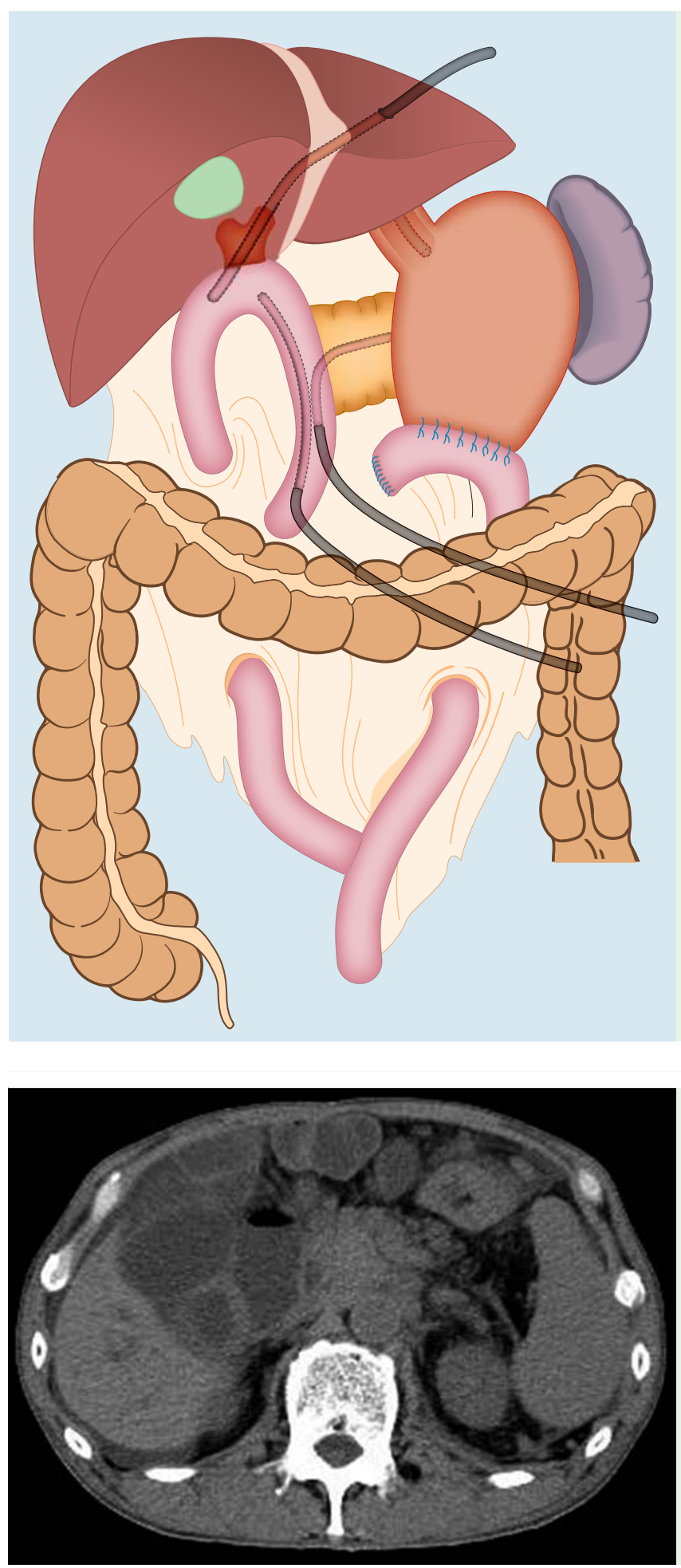

Fig. 1 Schematic of the Roux-en-Y reconstruction.

Fig. 2 Computed tomography (CT) scan showing malignant afferent-loop obstruction.
A 64-year old man was admitted to our hospital because of acute cholangitis. He had previously undergone pancreatoduodenectomy with Roux-en-Y reconstruction for a pancreatic neuroendocrine carcinoma ( Fig.1). A computed tomography (CT) scan revealed afferent-loop obstruction due to lymph node metastases (๑ Fig. 2).

A double-balloon enteroscope (DBE; EN450T5/W; Fujifilm Medical Systems) was inserted near the stenosis of the afferent loop, from where it was just possible to pass the enteroscope through the stenosis ( Video 1). A clip was placed on either side of the stenosis and contrast medium was also injected to identify the stenosis ( Fig. 3a). The overtube was moved back from the stenosis to take a distance for stent delivery and was fixed by the balloon. The enteroscope was then removed leaving the guidewire (0.035-inch Jagwire; Boston Scientific, Tokyo, Japan) in place.

A Niti-S D pyloric/duodenal stent $(22 \mathrm{~mm} \times$ $6 \mathrm{~cm}$; TaeWoong Medical, Seoul, South Korea) was advanced along the guidewire via the overtube ( Fig.3b). The stent delivery system was passed easily through the end of the overtube. The self-expanding metal stent (SEMS) was delivered under fluoroscopic guidance using the contrast medium and the clips as landmarks ( $\bullet$ Fig. $3 \mathrm{c}$ ). After the SEMS had been placed, the enteroscope was inserted again via the overtube to confirm that the SEMS was correctly positioned. The patient's cholangitis was successfully controlled by placement of the SEMS.

SEMS placement for malignant smallbowel obstruction is challenging because it is sometimes difficult to approach the stenosis, and it is not possible to pass a stent delivery system through the working channel of an enteroscope. A few reports of the use of an endoscopic approach exist, but none of these have included malignant

\section{Video 1}

A double-balloon enteroscope was inserted close to the afferent-loop obstruction. After the stenosis had been identified endoscopically and fluoroscopically, the enteroscope was carefully removed. The stent delivery system was advanced over the guidewire via the overtube and an enteral stent was delivered under fluoroscopic guidance. 

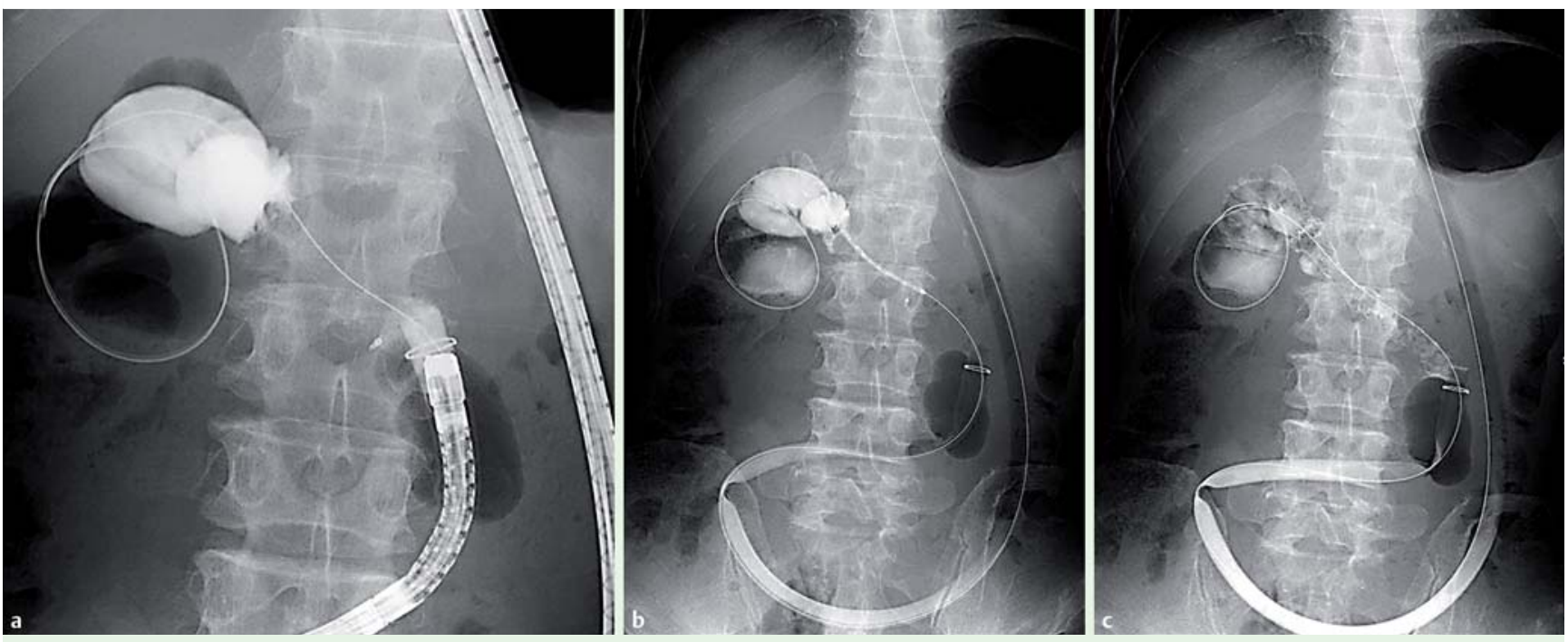

Fig. 3 Fluoroscopic views showing: a malignant afferent-loop obstruction; $\mathbf{b}$ the stent delivery system being introduced via the overtube; $\mathbf{c}$ the enteral stent in place.

afferent-loop obstruction following Rouxen-Y reconstruction [1-4]. SEMS placement via percutaneous transhepatic biliary drainage has been reported, but it carries a risk of biliary leakage [5]. In this case, it was possible to securely deliver the SEMS to the stenosis because the overtube was firmly fixed by the balloon. Therefore, DBE-assisted SEMS placement is a useful procedure in the management of malignant afferent-loop obstruction.

\section{Endoscopy_UCTN_Code_TTT_1AP_2AD}

\section{Competing interests: None}

Takashi Sasaki', Hiroyuki Isayama', Hirofumi Kogure ${ }^{1}$, Atsuo Yamada ${ }^{1}$, Taku Aoki ${ }^{2}$, Norihiro Kokudo², Kazuhiko Koike ${ }^{1}$

${ }^{1}$ Department of Gastroenterology, Graduate School of Medicine,

The University of Tokyo, Tokyo, Japan

${ }^{2}$ Hepato-Biliary-Pancreatic Surgery Division, Department of Surgery, Graduate School of Medicine, The University of Tokyo, Tokyo, Japan

\section{References}

1 Ross AS, Semrad C, Waxman I et al. Enteral stent placement by double balloon enteroscopy for palliation of malignant small bowel obstruction. Gastrointest Endosc 2006; 64: 835-837

2 Lennon AM, Chandrasekhara V, Shin EJ et al. Spiral-enteroscopy-assisted enteral stent placement for palliation of malignant small-bowel obstruction (with video). Gastrointest Endosc 2010; 71: 422-425

3 Lee H, Park JC, Shin SK et al. Preliminary study of enteroscopy-guided, self-expandable metal stent placement for malignant small bowel obstruction. J Gastroenterol Hepatol 2012; 27: 1181-1186
4 Popa D, Ramesh J, Peter S et al. Small bowel stent-in-stent placement for malignant small bowel obstruction using a balloonassisted overtube technique. Clin Endosc 2014; 47: 108-111

5 Laasch HU. Obstructive jaundice after bilioenteric anastomosis: transhepatic and direct percutaneous enteral stent insertion for afferent loop occlusion. Gut Liver 2010; 4 (Suppl. 01): 589-595

\section{Bibliography}

DoI http://dx.doi.org/

10.1055/s-0034-1377633

Endoscopy 2014; 46: E541-E542

(c) Georg Thieme Verlag KG

Stuttgart · New York

ISSN 0013-726X

\section{Corresponding author}

\section{Takashi Sasaki, MD, PhD}

Department of Gastroenterology

Graduate School of Medicine

The University of Tokyo

7-3-1 Hongo, Bunkyo-ku

Tokyo 113-8655

Japan

sasakit-tky@umin.ac.jp 